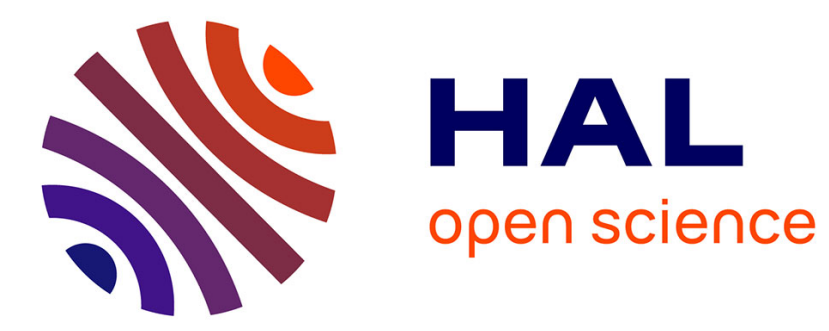

\title{
Magnetic coupling among spinel iron oxide microparticles by Mössbauer spectroscopy
}

E. Tronc, D. Bonnin

\section{To cite this version:}

E. Tronc, D. Bonnin. Magnetic coupling among spinel iron oxide microparticles by Mössbauer spectroscopy. Journal de Physique Lettres, 1985, 46 (10), pp.437-443. 10.1051/jphyslet:019850046010043700 . jpa-00232541

HAL Id: jpa-00232541

https://hal.science/jpa-00232541

Submitted on 1 Jan 1985

HAL is a multi-disciplinary open access archive for the deposit and dissemination of scientific research documents, whether they are published or not. The documents may come from teaching and research institutions in France or abroad, or from public or private research centers.
L'archive ouverte pluridisciplinaire HAL, est destinée au dépôt et à la diffusion de documents scientifiques de niveau recherche, publiés ou non, émanant des établissements d'enseignement et de recherche français ou étrangers, des laboratoires publics ou privés. 
Classification

Physics Abstracts

$76.80-75.60 \mathrm{~J}-75.30 \mathrm{G}-75.30 \mathrm{~K}$

\title{
Magnetic coupling among spinel iron oxide microparticles by Mössbauer spectroscopy
}

\author{
E. Tronc \\ Chimie de la Matière Condensée (*), ENSCP, 11, rue Pierre-et-Marie Curie, 75231 Paris Cedex 05, \\ France \\ and D. Bonnin
}

Physique Quantique, ESPCI, 10, rue Vauquelin, 75231 Paris Cedex 05, France

(Reçu le 11 décembre 1984, accepté le 29 mars 1985)

\begin{abstract}
Résumé. - Les spectres Mössbauer de colloïdes d'oxyde de fer hydraté de type spinelle et leur évolution en fonction du traitement thermique ont été interprétés à partir d'un modèle dérivé du modèle de champ moyen de Weiss. On déduit que les interactions entre les particules l'emportent sur l'énergie d'anisotropie des particules isolées. L'existence d'une texture cristalline au sein d'agrégats est suggérée.

Abstract. - Mössbauer spectra of hydrous spinel iron oxide colloids and their evolution with thermal treatment have been interpreted using a modified Weiss local field model. It is found that interparticle interactions overcome the single particle anisotropy energy. Existence of a crystalline texture within aggregates is suggested.
\end{abstract}

Mössbauer spectra of magnetic microcrystals are usually interpreted (see reviews [1,2]) in terms of non interacting particles with thermal fluctuations of their magnetization vector (M) among the easy directions of magnetization (superparamagnetic relaxation, SR) or about an easy direction in a pre-relaxation process (collective magnetic excitations, $\mathrm{CME}$ ). Uniaxial magnetic anisotropy is generally assumed so that the anisotropy energy is taken as :

$$
E(\theta)=K V \sin ^{2} \theta \quad(K>0)
$$

where $K$ is the anisotropy energy constant, $V$ is the particle volume and $\theta$ is the angle between $\mathbf{M}$ and the symmetry axis. $K$ values thus obtained are much larger than bulk magnetocrystalline data, and size dependent, what is attributed to surface effects. Corresponding models applied to patterns of hydrous spinel iron oxide colloids worked correctly except for particles about $8 \mathrm{~nm}$ in size, at room temperature. It led us to account explicitly for magnetic coupling between the particles. This is reported here.

Materials were obtained by alkalizing mixtures of $\mathrm{FeCl}_{2}$ and $\mathrm{FeCl}_{3}$ by soda (samples $\mathrm{A}, \mathrm{B}, \mathrm{C}$ ) or ammonia (samples D, E) and drying the precipitate in an evacuated dessicator with $\mathrm{P}_{2} \mathrm{O}_{5}$.

(*) Laboratoire associé au CNRS, no 302. 
Detailed preparation conditions and physico-chemical properties have been already reported [3-5]. All materials were of a defect spinel-type [6] with lattice constant $(0.835 \mathrm{~nm})$ in between that of $\mathrm{Fe}_{3} \mathrm{O}_{4}$ and that of $\gamma-\mathrm{Fe}_{2} \mathrm{O}_{3}$, the $\mathrm{Fe}^{2+}$ concentration determined by chemical analysis was always less than 0.10 . Size distributions were evaluated from electron micrographs and fitted to Log-normal laws :

$$
f(D)=(\sqrt{2 \pi} \sigma D)^{-1} \exp \left[-\left(\ln D / D_{0}\right)^{2} / 2 \sigma^{2}\right]
$$

where the median diameter $D_{0}$ and the standard deviation $\sigma$ were the two adjustable parameters. Sizes $D_{\mathrm{x}}$ evaluated from X-ray diffraction (XRD) line widths using the Scherrer method were coherent with these distributions. We found $D_{\mathrm{x}} \sim D_{0} \exp \left(2.5 \sigma^{2}\right.$ ) (ratio of the third to second moment of $f(D))$ in agreement with the meaning of $D_{\mathbf{x}}$. Moderate thermal treatments $(24 \mathrm{~h})$ were performed. XRD profiles proved that the size of the spinel domains scattering coherently was not altered even when partial conversion to $\alpha-\mathrm{Fe}_{2} \mathrm{O}_{3}$ had occurred, and that $\alpha$-domains were always much larger than the spinel ones. All observations about the $\gamma \rightarrow \alpha$ transformation, its spreading over a temperature range, size and preparation dependent, agreed with known features [7-8].

Mössbauer spectra were recorded with a conventional spectrometer operating in the constant acceleration mode using a ${ }^{57} \mathrm{Co} / \mathrm{Rh}$ source. Absorbers, with a thickness in the range $10-15 \mathrm{mg} \mathrm{Fe} /$ $\mathrm{cm}^{2}$ were made up of powders embedded in an acrylic resin. Dilution in the resin was checked to have no effect on the spectra. Typical patterns are given in figure 1. Large particles gave a pattern identical to that of $\gamma-\mathrm{Fe}_{2} \mathrm{O}_{3}$ [9]. The size effect prominent at room temperature (RT) was much weakened on cooling giving more or less asymmetrically broadened six-line patterns (Fig. 2A) at $80 \mathrm{~K}(\mathrm{NT})$ and the spectrum of bulk $\gamma-\mathrm{Fe}_{2} \mathrm{O}_{3}$ at $4 \mathrm{~K}$. All patterns but one (Fig. 1C) could be correctly fitted using the SR-CME models [1, 10-12] based on the volume weighted size distributions $F(D)=D^{3} f(D)$, and assuming a single $K$ value per sample. Corresponding $K$ values were in the range $10^{4}-10^{5} \mathrm{~J} / \mathrm{m}^{3}$, increasing with decreasing particle size, and coherent with data already reported [13-15].

Thermal treatments induced no significant modification in the RT pattern of as-prepared Na containing materials (sample B) up to appearance of $\alpha-\mathrm{Fe}_{2} \mathrm{O}_{3}$, but progressive changes (Fig. 2) were observed for samples either prepared with ammonia (series D) or freed from $\mathrm{Na}^{+}$ions by acid treatment (series $\mathrm{A}, \mathrm{C}$ ) [5]. When involved, $\alpha-\mathrm{Fe}_{2} \mathrm{O}_{3}$ always gave a narrow-line XRD pattern

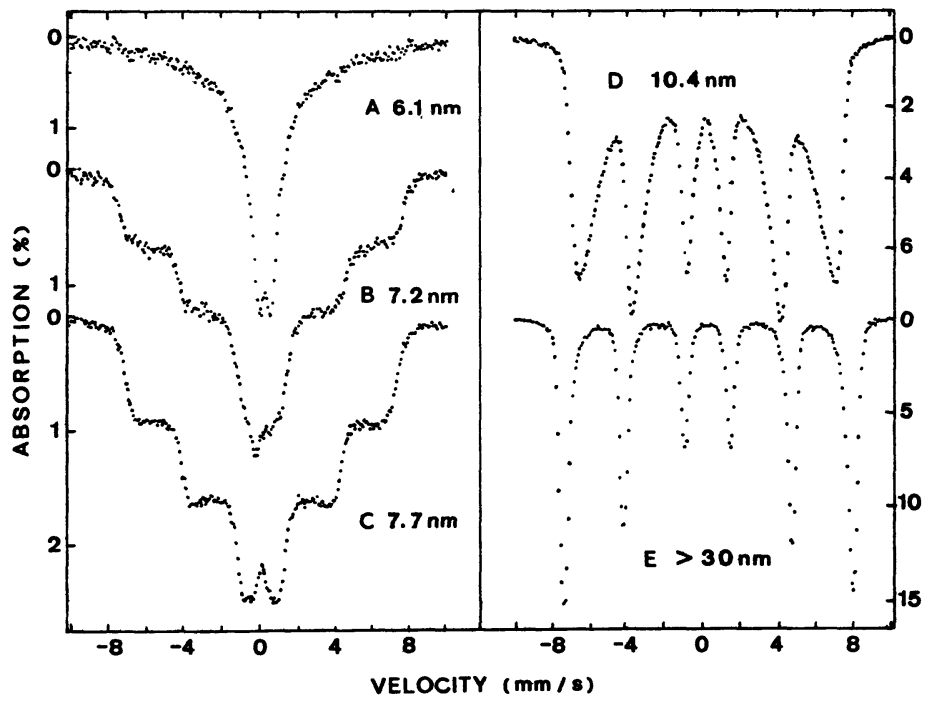

Fig. 1. - Mössbauer spectra of hydrous spinel iron oxide colloids with various sizes $\left(D_{\mathbf{x}}\right)$ at $295 \mathrm{~K}$. 
and a narrow-line RT Mössbauer sextuplet, indicating large particle sizes. Clearly it is absent from the materials studied here (Fig. 2). Size effect being ruled out by X-ray diffraction, the data evolution with the annealing tended to indicate an increase in $K$. Patterns with typical « udder »like shape (Fig. 2A1, A2, C1) proved inconsistent with the SR-CME model. Size dependence of $K$, release of the standard deviation of the size distribution, cubic instead of uniaxial anisotropy, produced no significant improvement in the fits. Such patterns with better resolution of the inner lines actually are in conflict with the SR-CME model which leads to a collapse of these lines first. They involve hyperfine field (HF) distributions which extend much lower than what is predicted $[16,17]$.

Reduced hyperfine fields may be connected with surface effects [1] such as progressive decoupling of surface spins from the core, or existence of a perturbed layer. The surface field may not be significantly smaller than that of the bulk at low temperature, but distinct temperature dependences may be thought of, leading to notable reduction at the surface, at room temperature. Evolution of the data with annealing may support the existence of a hydrous layer whose dehydration results in progressive ordering of surface spins, that ordering being hindered by $\mathrm{Na}^{+}$ions. No analysis has however been attempted in this way, mainly because independent characterization of the layer was lacking.

We have exploited an alternative to the HF reduction, which resides in magnetic interactions between the particles [18]. This viewpoint was supported by the existence of aggregates in solution [5] and by the well-known texture of polycrystalline acicular $\gamma-\mathrm{Fe}_{2} \mathrm{O}_{3}$ particles [19]. A few publications dealing with similar features in other materials have been reported recently. The $\mathrm{HF}$ distributions have been either computed from the spectra $[16,17]$ or adjusted by trial and error [20]. We have adopted another way.

Following Mørup's paper [18], magnetic interactions between the particles were taken into account using a modified Weiss mean field model. Within the approximations that all particles are nearly identical with nearly identical surroundings, and ignoring time dependent phenomena, average values can be used. Then the anisotropy energy due to interaction between particle $i$ with magnetization $\mathbf{M}_{i}$ and its surroundings may be written :

$$
E_{i}=-\mathbf{H}_{\mathrm{m}} \cdot \mathbf{M}_{\boldsymbol{i}}
$$

where $\mathbf{H}_{\mathrm{m}}$ is the mean field proportional to the average magnetization $\langle\mathbf{M}\rangle$ of the $n_{i}$ neighbouring particles. Assuming for simplicity that $H_{\mathrm{m}}$ is parallel to the anisotropy axis of the crystallite, the anisotropy energy (1) for particle $i$ becomes :

$$
E(\theta)=K V \sin ^{2} \theta-H_{\mathrm{m}} M_{\mathrm{s}} V \cos \theta
$$

where $M_{\mathrm{s}}$ is the saturation magnetization per unit volume. This is formally equivalent to the energy of an isolated particle in the presence of an external field applied along the easy direction.

Neglecting any dynamical effect, the probability that the direction of $\mathbf{M}_{i}$ is in the range $(\theta$, $\theta+\mathrm{d} \theta)$ is :

$$
p(\theta) \mathrm{d} \theta=\exp [-E(\theta) / k T] \sin \theta \mathrm{d} \theta / \int_{0}^{\pi} \exp [-E(\theta) / k T] \sin \theta \mathrm{d} \theta
$$

where $k$ is the Boltzmann constant and $T$ is the temperature. Hence, since the magnetic hyperfine field is proportional to magnetization, as far as the correlation time of the thermal fluctuations is short compared to the Larmor precession period $\tau_{\mathrm{N}}$ of the nuclear moment (typically a few $10^{-9} \mathrm{~s}$ ), the magnetic splitting of component $i$ can be expressed in terms of an average hyperfine field given by :

$$
H_{\mathrm{obs}}=H_{0}\langle\cos \theta\rangle
$$


where $H_{0}$ is the hyperfine field in the absence of fluctuations and $\langle\cos \theta\rangle$ is the thermal average of $\cos \theta$.

- For $h=H_{\mathrm{m}} M_{\mathrm{s}} / 2 K \geqslant 1$, i.e. for dominant interaction anisotropy, $E(\theta)$ has one minimum at $\theta=0$ and $\langle\cos \theta\rangle$ is given by [21] :

$$
\begin{aligned}
\langle\cos \theta\rangle & =\int_{0}^{\pi} \exp [-E(\theta) / k T] \cos \theta \sin \theta \mathrm{d} \theta / \int_{0}^{\pi} \exp [-E(\theta) / k T] \sin \theta \mathrm{d} \theta \\
& =[1-\exp (-4 \alpha h)] /\left\{2 \sqrt{\alpha}\left[y\left(\alpha_{1}\right)+\exp (-4 \alpha h) y\left(\alpha_{2}\right)\right]\right\}-h
\end{aligned}
$$

with

$$
\alpha=K V / k T \quad \alpha_{1}=\sqrt{\alpha}(1+h) \quad \alpha_{2}=\sqrt{\alpha}(1-h) \quad y(x)=\int_{0}^{x} \exp \left(t^{2}-x^{2}\right) \mathrm{d} t .
$$

- For $h<1, E(\theta)$ has minima at $\theta=0\left(W_{1}\right)$ and $\theta=\pi\left(W_{2}\right)$ separated by a maximum at $\theta_{\mathrm{m}}=\cos ^{-1}(-h)$, and the direction of $\mathbf{M}_{i}$ may fluctuate between the two wells. Because of the asymmetrical energy barrier two relaxation times are involved $[1,10]$. At the view of the breadth of the experimental size distributions, a simplified model has been used. Depending on the ratio $W_{i j} / k T=\alpha_{i}^{2}(i=1, j=2$ and $i=2, j=1)$ where $W_{i j}$ is the energy barrier from $W_{i}$ to $W_{j}$, three limiting cases have been considered :

1) $1 \ll \alpha_{2}^{2}$. Since $h$ is less than 1 , it necessarily corresponds to $K V / k T \gg 1$. Relaxation in either way may be neglected. Two distinct populations are implied. The probability $p_{1}$ that the direction of $\mathbf{M}_{i}$ is in the range $\left(0, \theta_{\mathrm{m}}\right)$ is from (5):

$$
p_{1}=y\left(\alpha_{1}\right) /\left[y\left(\alpha_{1}\right)+\exp (-4 \alpha h) y\left(\alpha_{2}\right)\right] .
$$

The corresponding average of $\cos \theta$ is :

$$
\left\langle\cos \theta_{1}\right\rangle=\left[1-\exp \left(-\alpha_{1}^{2}\right)\right] /\left[2 \sqrt{\alpha} y\left(\alpha_{1}\right)\right]-h .
$$

For the range $\left(\theta_{\mathrm{m}}, \pi\right)$ we have $p_{2}=1-p_{1}$ and :

$$
\left\langle\cos \theta_{2}\right\rangle=-\left[1-\exp \left(-\alpha_{2}^{2}\right)\right] /\left[2 \sqrt{\alpha} y\left(\alpha_{2}\right)\right]-h .
$$

2) $\alpha_{2}^{2}<1 \ll \alpha_{1}^{2}$. Intermediate values of $K V / k T$ are concerned, the larger $h$, the wider their range. Relaxation proceeds preferentially from $W_{2}$ to $W_{1}$. Hence neglecting the transient effects, one may consider the range $\left(0, \theta_{\mathrm{m}}\right)$ only and $\langle\cos \theta\rangle$ is given by (9).

3) $\alpha_{1}^{2}<1$. Small $K V / k T$ are involved. Time dependent effects are prominent. Brown's low energy barrier approximation [10] involving only one relaxation time $\tau$ may be used. If $\tau \ll \tau_{N}$, the thermal average approximation is still valid and $\langle\cos \theta\rangle$ is again given by (7), otherwise the Mössbauer component may be calculated according to [12].

For $\alpha<0.1, \cos \theta(7)$ is well approximated by the Langevin function $L(\beta)=\operatorname{coth} \beta-1 / \beta$ with $\beta=2 \alpha h=H_{\mathrm{m}} M_{\mathrm{s}} V / k T$. For high $x(x>6), y(x) \simeq x /\left(2 x^{2}-1\right)$ leading to Mørup's approximation [18] $\langle\cos \theta\rangle \simeq 1-1 /(2 \alpha+\beta)$. In the intermediate range, $y(x)$ may be developed into convergent series, nevertheless we found it more convenient to compute it by linear interpolation between tabulated data previously computed using the Romberg method. Effects at the boundaries between cases 1, 2, 3 were assumed to involve small fractions of the size distributions only, they were neglected. For sake of computing efficiency, calculation of the relaxation time $\tau$ was omitted and thermal averages were considered only.

This model has been applied to size distributions $F(D)$ corresponding to non-heated materials (A, C, D) and truncated at the minimum and maximum experimental $D$ values, with 50 points in between at least. Hyperfine parameters of bulk material were taken as obtained from large par- 
ticles (Fig. 1E). The quadrupole splitting observed for superparamagnetic materials (Fig. 1A) and responsible for the slight asymmetry of the central part of some spectra (Fig. 1B) was further ignored. The elementary Lorentzian line-width was fixed at 0.5 and $0.8 \mathrm{~mm} / \mathrm{s}$ for NT and RT data fits respectively. The patterns were least-squares fitted with two adjustable parameters, $K$ and $H_{\mathrm{m}} M_{\mathrm{s}}$, at maximum. For sample $\mathrm{A}_{1}, H_{\mathrm{m}} M_{\mathrm{s}}$ was the dominant factor with ambiguity on $K$ attributed to the small $\beta$ 's involved (the case of sample A, obviously dominated by fast superparamagnetic relaxation at room temperature was not treated). For series $\mathrm{D}$, notable correlations appeared, the patterns mainly depending on $2 K+H_{\mathrm{m}} M_{\mathrm{s}}=2 K_{\mathrm{eq}}$. NT spectra were fitted with the only parameter $2 K_{\text {eq }}$. Corresponding results are given in table I and figure 2 . They show that $h \gtrsim 1$ for all the samples, i.e. the inter-particle interactions overcome the single particle anisotropy energy. The three limiting cases discussed above $(h<1)$ therefore seem not to be relevant for the present samples.

Table I. - Anisotropy energy parameters.

\begin{tabular}{|l|rrr|rr|c|}
\hline \multirow{2}{*}{ Sample ( $\left.{ }^{1}\right)$} & \multicolumn{3}{|c|}{ Size distribution } & $K\left({ }^{2}\right)$ & $H_{\mathrm{m}} M_{\mathrm{s}}\left({ }^{2}\right)$ & $\left.2 K_{\mathrm{eq}}{ }^{3}\right)$ \\
& $D_{0}(\mathrm{~nm})$ & $\sigma$ & $D_{\mathbf{x}}(\mathrm{nm})$ & $\left(10^{3} \mathrm{~J} / \mathrm{m}^{3}\right)$ & $\left(10^{3} \mathrm{~J} / \mathrm{m}^{3}\right)$ \\
\hline $\mathrm{A}$ & 4.7 & 0.3 & 6.1 & & & 116 \\
$\mathrm{~A}_{1}(433 \mathrm{~K})$ & & & & $2-20$ & 35 & 143 \\
$\mathrm{~A}_{2}(473 \mathrm{~K})$ & & & & 3 & 86 & 168 \\
\hline $\mathrm{C}_{(}(433 \mathrm{~K})$ & 6.0 & 0.32 & 7.7 & 12 & 23 & 59 \\
$\mathrm{C}_{2}(523 \mathrm{~K})$ & & & & 4 & 54 & 79 \\
\hline $\mathrm{D}$ & & & & 5 & 74 & 83 \\
$\mathrm{D}_{1}(433 \mathrm{~K})$ & 8.2 & 0.31 & 10.4 & \multicolumn{2}{|c|}{44} & 44 \\
\hline
\end{tabular}

( $\left.{ }^{1}\right)$ Annealing temperature given in brackets (annealing time $\sim 24 \mathrm{~h}$ ).

$\left({ }^{2}\right)$ Values deduced from data recorded at $295 \mathrm{~K}$. For series D, due to high correlations between $K$ and $H_{\mathrm{m}} M_{\mathrm{s}}$, the only significant parameter is $2 K_{\mathrm{eq}}=2 \mathrm{~K}+H_{\mathrm{m}} M_{\mathrm{s}}$.

$\left({ }^{3}\right)$ Values deduced from data recorded at $80 \mathrm{~K}$.

Interpreting these spectra probably is not unequivocal. However the quality of the fits, the consistency in the parameter values along with the reduced number of adjusted parameters, in spite of the drastic approximations used, reasonably support our view. Interactions were considered as an intrinsic feature of the materials. They likely are of magnetostatic and exchange types. Taking $M_{\mathrm{s}}=0.5 \mathrm{~Wb} / \mathrm{m}^{2}$ as in bulk $\gamma-\mathrm{Fe}_{2} \mathrm{O}_{3}[19]$ overall anisotropy fields $H_{\mathrm{a}}=2 K_{\mathrm{eq}} / M_{\mathrm{s}}$ in the range $80-340 \mathrm{kA} / \mathrm{m}$ are deduced. Increasing interactions within a series probably correspond to gradual particle sintering caused by progressive escape of species $\left(\mathrm{H}_{2} \mathrm{O}, \mathbf{N H}_{3}\right)$ rather weakly bonded to the colloids. Annealing-independent spectra for Na-containing materials support a strong bonding for $\mathrm{Na}^{+}$ions, and an anti-sintering effect. Significant mismatch between values of $2 K_{\text {eq }}$ at $295 \mathrm{~K}$ and $80 \mathrm{~K}$ is obtained for series A and C (Table I). It decreases with increasing $H_{\mathrm{m}} \boldsymbol{M}_{\mathrm{s}}$ within a series, or with increasing sizes. This logically indicates that the discrepancy originates in a reduction of $\langle\mathbf{M}\rangle$ at $295 \mathrm{~K}$ caused by significant fluctuations of the magnetization vector of the various particles.

It seems that thermal treatment or increasing size stabilizes $K$ at about $4 \times 10^{3} \mathrm{~J} / \mathrm{m}^{3}$. Then, comparison with magnetocrystalline constant $K_{1}\left(-4.7 \times 10^{3} \mathrm{~J} / \mathrm{m}^{3}\right)$ of $\gamma-\mathrm{Fe}_{2} \mathrm{O}_{3}[19]$ supports 

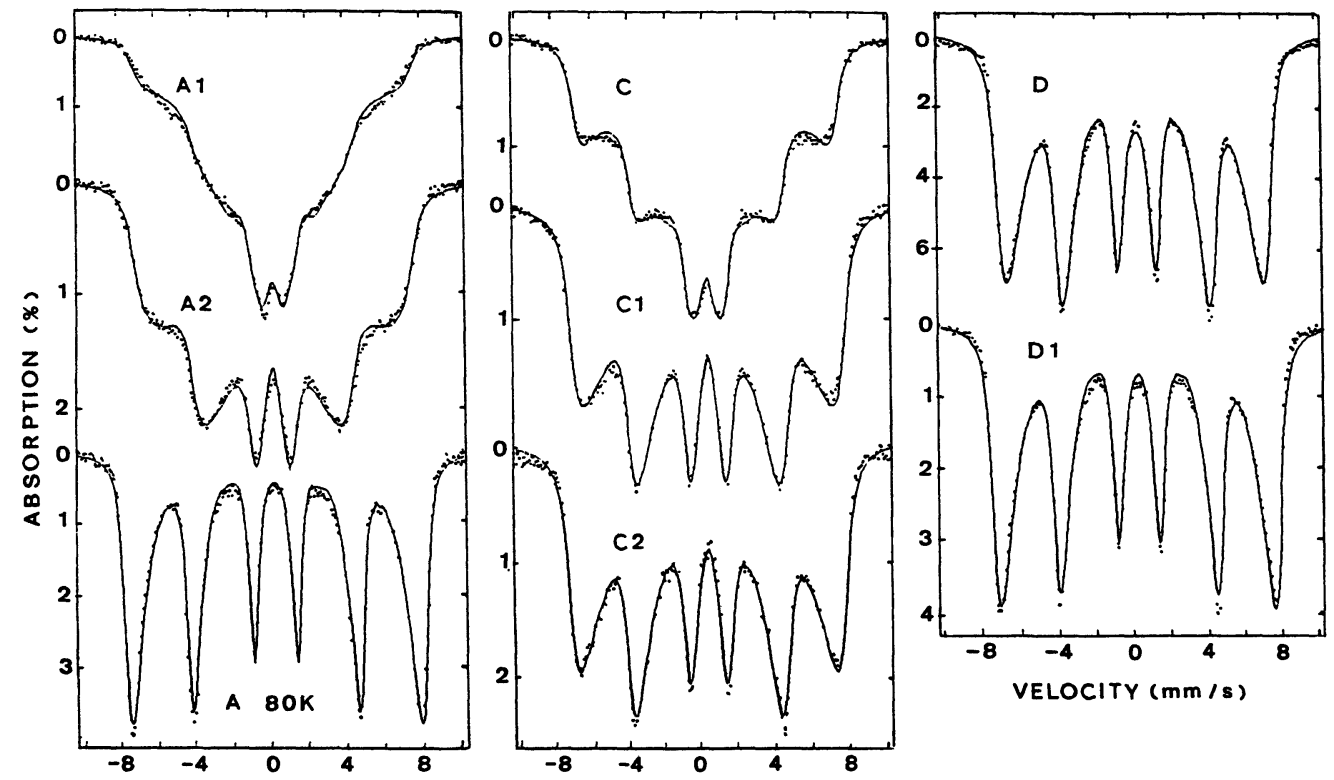

Fig. 2. - Experimental and theoretical Mössbauer spectra of $\mathrm{Na}$-free spinel iron oxide colloids as a function of thermal treatment. All spectra but one $(\mathrm{A}, 80 \mathrm{~K})$ taken at $295 \mathrm{~K}$, annealing conditions given in table I. Theoretical patterns computed using the modified Weiss mean field model developed in the text.

the idea of easy direction of magnetization [22] along [110] axis of the cubic cell as in acicular $\gamma-\mathrm{Fe}_{2} \mathrm{O}_{3}$ particles. It suggests the existence of a crystalline texture within aggregates and may justify the assumption of the magnetic texture, of the super-ferromagnetic (or antiferromagnetic) type [16] implied by the model as used. Support to a crystalline texture may be given by typical features of the $\gamma \rightarrow \alpha \mathrm{Fe}_{2} \mathrm{O}_{3}$ transformation which systematically produces $\alpha$-domains much larger than the spinel ones [7]. One may think that shrinking of « super-ferromagnetic crystalloids " governs the transformation. Further experimental support is obviously needed. An electron microscopic study is planned that way.

It will be shown elsewhere [23] that most of the behaviour of the hydrous spinel colloids may actually be understood using the crystalloid [24] concept, with insertion, de-insertion and exchange reactions in the inter-crystallite space. This view likely applies to other colloidal materials. Various systems are known to give dispersions where ordered aggregates form [24] whose swelling and shrinking may be produced by proper variation of the medium. Then even in flocculates, some texture may be expected. If magnetic order exists within the particles, a magnetic texture (more or less long-ranged) is likely to occur. Most of the iron oxy-hydroxyde colloids may actually be concerned. This is already true for $\alpha$-FeOOH [16], and presumable for $\delta$-FeOOH [25].

Apart from this, the modified Weiss mean field model that we have used may apply to other closely-packed fine grained materials. Good candidates have already appeared [17, 26].

\section{Acknowledgments.}

We wish to express our thanks to R. Massart and J. P. Jolivet (University Paris VI) for providing us with the various samples. 


\section{References}

[1] M $\phi$ RuP, S., Dumesic, J. A. and Tops $\phi$ E, H., Applications of Mössbauer Spectroscopy, R. L. Cohen Ed. (Academic Press, New York) 1980, vol. 2, p. 1.

[2] Dormann, J. L., Rev. Phys. Appl. 16 (1981) 275.

[3] Massart, R., Brevet Français, 7918842 (1979); US Patent 4329241 (1982).

[4] Massart, R., IEEE Trans. Mag. MAG-17 (1981) 1247.

[5] Jolivet, J. P., Massart, R. and Fuchart, J. M., Nouv. J. Chim. 7 (1983) 325.

[6] Tronc, E., Jolivet, J. P. and Massart, R., Mater. Res. Bull. 17 (1982) 1365.

[7] Feitknecht, W. and Mannweller, U., Helv. Chim. Acta 50 (1967) 570.

[8] Bando, Y., Kiyama, Y., Takada, T. and KaChI, S., Japan J. Appl. Phys. 4 (1965) 240.

[9] HANEDA, K. and Morrish, A. H., Solid State Commun. 22 (1977) 779.

[10] Brown Jr., W. F., Phys. Rev. 130 (1963) 1677.

[11] Aharoni, A., Phys. Rev. A 135 (1964) 447.

[12] Wickman, H. H., Klein, M. P. and ShiRley, D. A., Phys. Rev. 152 (1966) 345.

[13] Haneda, K. and Morrish, A. H., Phys. Lett. A 64 (1977) 259.

[14] Coey, J. M. D. and Khalafalla, D., Phys. Status Solidi A 11 (1972) 229.

[15] Boudart, M., Delbouille, A., Dumesic, J. A., Khammouma, S. and Tops $\phi$ E, H., J. Catal. 37 (1975) 486.

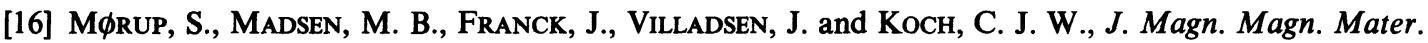
40 (1983) 163.

[17] Vandenberghe, R. E., Vanleerberghe, R. and Gryffroy, D., Phys. Lett. A 101 (1984) 297.

[18] MфRUP, S., J. Magn. Magn. Mater. 37 (1983) 39.

[19] BATE, G., Ferromagnetic Materials, E. P. Wohlfarth Ed. (North-Holland, Amsterdam) 1980, vol. 2, p. 381 .

[20] Tamura, I. and Hayashi, M., Surface Sci. 146 (1984) 501.

[21] West, F. G., J. Appl. Phys. S 32 (1961) 2495.

[22] Herpin, A., Théorie du Magnétisme (PUF, Paris) 1968, p. 334.

[23] Tronc, E., Bonnin, D., Jolivet, J. P. and Massart, R., to be published.

[24] Heller, W., Polymer Colloids, E. Fitch Ed. (Plenum Press, New York) 1980, vol. 2, p. 153.

[25] Povitskit, V. A., Makarov, E. F., Murashko, N. V. and Salugin, A. N., Phys. Status Solidi a 33 (1976) 783.

[26] CUI, J. R., Thesis (University, Paris VII), 1984. 